\title{
Napoleone III - più che la guerra poterono i calcoli
}

\section{di Luciano Sterpellone}

Quale migliore ragione per Napoleone III per dichiarare guerra alla Prussia? Il crescere della potenza prussiana aveva accentuato i timori del II Impero francese, aggravati dallo spettro di una candidatura degli Hohenzollern al trono di Spagna.

Oggi ci appaiono questioni anacronistiche, forse assurde: ma se siamo avvezzi a farci guerra per il petrolio, non si vede perché i nostri avi non dovessero farla per questioni dinastiche.

Comunque, il 19 luglio 1870 Napoleone III (Luigi Bonaparte), nipote di Napoleone I, dichiarò guerra alla Prussia. La guerra franco-prussiana sarebbe durata dieci mesi, ma i combattimenti veri e propri si sarebbero svolti solo nel corso di sei mesi. Come spesso accade, l'attaccante fece male i conti circa le reali forze del nemico: tant'è che con precisione teutonica e con una rapidità imprevista tre eserciti prussiani invasero in poco tempo la Francia; e a Sedan, sulle rive della Mosa, dopo uno snervante assedio, il $1^{\circ}$ settembre fu ingaggiata una furibonda battaglia: 120.000 soldati francesi si trovarono di fronte un esercito bene armato e forte di 220.000 prussiani perfettamente addestrati.

\section{Meglio morire...}

La battaglia fu sanguinosa, con atti di eroismo e di coraggio da ambo le parti, con l'Imperatore che si spostava instancabilmente da una schiera all'altra dei suoi incurante delle pallottole. Per incitarli, per abnegazione, per dare l'esempio? "Macché - affermano gli storici in base a prove testimoniali -: solo perché non ne poteva più di una violenta colica renale che (complici i prussiani?...) era sopravvenuta proprio nel momento sbagliato".

Un calcolo vescicale che lo tormentava già da quattordici anni, ma che ora si faceva intempestivamente sentire proprio nel bel (si fa per dire) mezzo della battaglia.

Non era certo la prima volta che ne soffriva. Sin'allora, contro le coliche nulla avevano potuto i medici con i risibili mezzi dei quali disponevano, quasi tutti basati su infusi di erbe: cipolla, finocchio, ortica, gelso, gra- migna, prezzemolo, erica, ippocastano, rosa canina, angelica, ginepro, levisco, anche ciliegie. In alternativa, medicamenti a base di zinco, antimonio, giusquìmo, gusci d'uovo. Ma che non risolvevano nulla.

A Sedan, forse anche sotto lo stress del momento, il calcolo aveva ripreso a tormentare l'Imperatore: proprio come era stato cinquantacinque anni prima a Waterloo per il suo illustre predecessore a causa degli intensi dolori provocati dalle emorroidi: le abbondanti dosi di laudano invano somministrate gli avevano tolto ogni lucidità mentale per poter modificare all'istante le tattiche della battaglia. Ora i dolori al bacino provocati

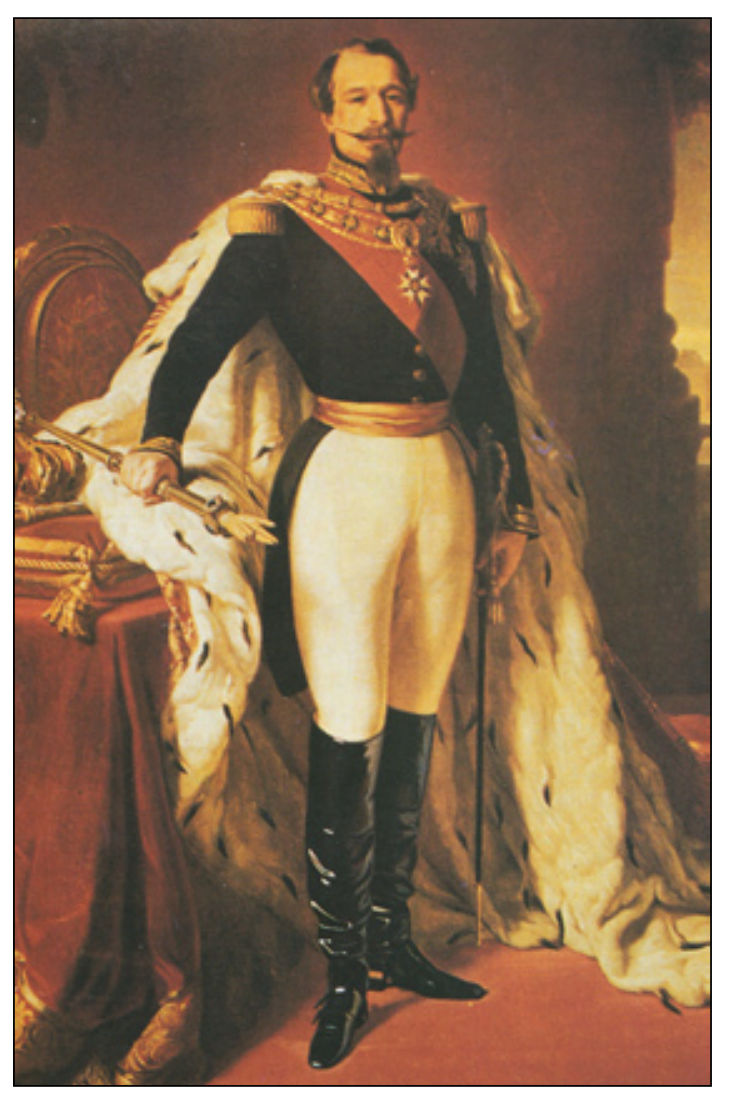

Napoleone III. Ritratto di F.X. Winterhalter, 1855 (Museo Napoleonico, Roma). 
dal calcolo erano così acuti, che per molti che lo videro in quelle condizioni egli si espose al fuoco nemico più per farla finita che per incitare i soldati.

Alla fine, verso sera, visto che le pallottole facevano di tutto per evitarlo, fece issare la bandiera bianca, e spedì un eloquente quanto cavalleresco messaggio all'imperatore Guglielmo di Prussia: «Sire, poiché non riesco a morire alla testa delle mie truppe, nulla mi resta che arrendermi e rimettere la mia spada nelle mani di Vostra Maestà».

Luigi Bonaparte e i suoi soldati furono presi tutti prigionieri. Poi, quando il $1^{\circ}$ marzo dell'anno successivo le truppe prussiane entrarono a Parigi, egli poté ricongiungersi alla moglie Eugenia che nel frattempo si era rifugiata dapprima presso il suo dentista americano a Parigi, e poi con questi a Chislehurst, nel Kent britannico.

Qui l'ex imperatore comincerà a sognare un rientro trionfale in Francia alla testa delle sue truppe: ma le reiterate ingravescenti coliche renali e le copiose ematurie gli faranno chiaramente capire che non è più il caso di accarezzare troppo certi sogni.

\section{Anche l'artrosi...}

I calcoli vescicali non erano il solo problema di salute che assillava "il piccolo imperatore". Ve n'erano altri, non meno fastidiosi, come le frequenti crisi di cefalea e - soprattutto - i "reumatismi". Le cronache parlano di "artrosi deformante", ma sull'esattezza tassonomica di questa diagnosi è lecito avanzare molte riserve alla luce delle moderne cognizioni.

Le "deformazioni" articolari - nel caso di Napoleone III localizzate soprattutto alle mani - possono essere state provocate da varie forme di artropatie, prima tra le quali l'artrite reumatoide, o forse anche - considerando la sua diatesi litiasica - un'artrite gottosa. Certo, la distinzione non è facile, data la confusione terminologica esistente al tempo anche riguardo alle reumopatie, che non di rado venivano comprese nell'unica denominazione di "gotta": termine questo invalso solo a partire dal XIII secolo, derivante dal latino gutta, in quanto si credeva che la malattia fosse dovuta a precipitazione "goccia a goccia" nelle articolazioni, di "umori corrotti”. Venne poi indicata come podagra, chiragra, gonagra, cleidagra, a seconda della localizzazione (piede, mano, ginocchio, spalla), e successivamente compresa nel grande gruppo delle artritidi sia acute che croniche, tutte connesse alla grande patologia umorale. Solo nell'Ottocento, la materia peccans degli antichi quale causa diretta della malattia fu "sostituita" con gli acidi, gli alcali e i sali.
Ma i medici del tempo non si preoccupavano troppo di certe sottigliezze... differenziali. Certo è che poco poté fare Napoleone III per le sue articolazioni, in quanto anche per le malattie articolari le "cure" del tempo erano quantomeno risibili, rappresentate per lo più da qualche preparato galenico e blando antidolorifico, dai salassi e dalla crenoterapia, dal cloruro d'ammonio, dal clorato di potassio, dallo ioduro di potassio, dall'oppio, nonché dai salassi e dall'idroterapia.

Quanto ai salassi il "razionale" (si fa per dire) era semplice: essendo i "reumatismi" ascritti a qualche difetto della qualità o del flusso (in greco reo $=$ scorro) degli umori nelle articolazioni, il solo modo per ristabilire la "crasi" e il libero flusso era la sottrazione di sangue onde allontanare gli umori "corrotti" e/o in eccesso. Purtroppo però di salassi se ne facevano a iosa, con gravi rischi per il paziente.

Relativamente alle cure idroterapiche (largamente usate anche contro i calcoli) ancora al tempo di Napoleone III la maggior parte dei medici vi faceva grande affidamento, specie se il paziente era benestante e aveva possibilità di raggiungere le fonti termali più rinomate. Particolarmente efficaci erano considerate, per le malattie reumatiche, le acque sulfuree, e arsenicatiferruginose, nonché quelle oligominerali. Ma spesso, più che non alle indicazioni specifiche, si badava alla risonanza della fonte e al prestigio che godeva, per cui specie ai ricchi e ai potenti piaceva molto peregrinare per le varie stazioni europee come status symbol e per potervi lasciare il proprio nome: senza risultati concreti per la patologia in atto, ma decisamente considerevoli per il benessere generale indotto al soggiorno in ambiente così sereno e salubre. Così, anche Napoleone III ha lasciato il segno della sua augusta presenza a Plombières-les-Bains, Vichy, Baden-Baden, Marienbad, oltre che a Calimanesti in Romania, e - in Italia - a Salsomaggiore e in altre stazioni.

Ciononostante, un tale convulso suo peregrinare per le più rinomate fonti europee, non giovò né alla sua litiasi urinaria né ai suoi disturbi articolari: semmai le terme di Plombières-les-Bains, sui Vosgi, più che non a lui avevano in precedenza giovato alla Francia, che con lo storico incontro ivi avvenuto il 20 luglio 1858 con Camillo Benso conte di Cavour aveva incamerato la Savoia in cambio dell'impegno di un appoggio militare francese in caso di aggressione del Regno Sardo da parte dell'Austria. Cabale d'altri tempi.

Cabale, anche perché certi accordi non si prendono tanto facilmente. Tant'è che Cavour, per demolire l'iniziale incertezza di Napoleone III su questo disegno (incertezza motivata dalla sua giustificata diffidenza verso l'Italia dopo essere sfuggito a ben tre attentati di matri- 
ce italiana!...), aveva preventivamente spedito a Parigi, oltre al grande diplomatico Costantino Nigra anche la propria infallibile arma segreta, la voluttuosa e procace contessa di Castiglione (Virginia Oldoini), non ancora ventenne. Costei non mancò di mettere tutta se stessa - è il caso di dirlo - nell'assolvere al meglio la missione affidatale. Del resto, le direttive ricevute personalmente da Cavour non ammettevano deroghe: «Usate tutti i mezzi che vi pare, ma riuscite!» .

E Virginia, senza preoccuparsi di smentire la purezza del proprio nome, riuscì a sedurre l'imperatore e a divenirne l'amante; magari non tanto segreta. Quando si dice l'amore di patria (?).

\section{Esilio con calcolo}

Dopo la battaglia di Sedan, la vittoria dei prussiani aveva cementato la loro alleanza con la Federazione tedesca con la costituzione del II Reich; Cancelliere fu nominato Otto von Bismark ("il cancelliere di ferro"). Tutto ciò preparava il campo alla I e poi alla II guerra mondiale. Un "campo" che poco prima era stato abbondantemente irrorato... dall'urina del decaduto Imperatore francese.

Nel suo sogno di un clamoroso ritorno al potere, Luigi Bonaparte poneva in primo piano un trionfale rientro a Parigi su di un fiero destriero bianco. L'11 dicembre 1872 , volle fare la prova generale, e montò in sella per andare da Chislehurst (dove si trovava in esilio) sino a Woolwich per visitare il figlio; ma dopo appena un chilometro fu costretto a scendere da cavallo non tanto per i dolori articolari alle mani e alla colonna vertebrale quanto per quelli ancora più tormentosi che erano insorti al perineo (evidentemente dovuti al calcolo vescicale), che gli impedivano di tenersi in sella. Già nel luglio dello stesso anno, dato l'aggravarsi delle coliche vescicali, erano giunti a Chislehurst due luminari londinesi, William Gull e Henry Thompson, per un consulto; l'augusto paziente era assistito dai medici personali Louis Conneau e Jean Nicolas Corvisart. Quest'ultimo era stato in precedenza il medico di Napoleone I, e dopo

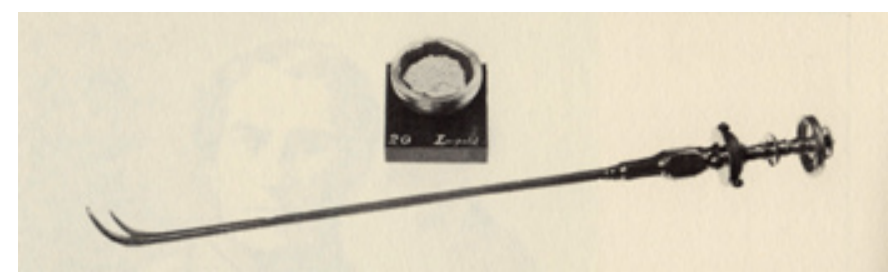

Litotrito impiegato da Thompson, e residuo del calcolo di Napoleone III, rimasto praticamente intatto (Hunterian Museum, Londra). la caduta dell'Impero aveva rinunciato a incarichi prestigiosi per rimanere fedele alla memoria del suo augusto paziente. Per tanti anni era rimasto nell'ombra, per riprendere improvvisamente quota dopo aver riscoperto il lavoro che cinquant'anni prima il francese Hyacinthe T. Laënnec aveva pubblicato sulla sua rivoluzionaria invenzione dello stetoscopio e sulla tecnica dell'“auscultazione mediata". Dopo aver tradotto in francese (dal latino) il lavoro di Laënnec, Corvisart aveva sottoposto a verifica i suoi risultati e definitivamente consacrato con la propria autorità la validità del metodo.

Fu proprio per suo merito che lo stetoscopio cominciò da allora a diffondersi in tutta l'Europa come strumento indispensabile per la diagnosi.

Pur essendo assistito da un medico di questa portata, Napoleone III rifiutò di essere sottoposto a sondaggio vescicale, l'unico metodo del tempo (la scoperta dei raggi $\mathrm{X}$ sarebbe avvenuta tredici anni dopo) per accertare la presenza di un calcolo: questa veniva denunciata dal brusco contatto del calcolo stesso contro il metallo della sonda; non consentiva tuttavia di valutarne con esattezza le dimensioni e la forma.

La situazione cominciò sempre più a peggiorare, sinché nel dicembre l'intervento apparve non più procrastinabile: compariva ematuria al minimo sforzo, l'urina appariva densa, piena di pus e di depositi, e il paziente avvertiva un dolore intenso e continuo al basso ventre e al perineo. Così, in via preliminare, il giorno dopo il Natale 1872, il paziente fu sottoposto ad anestesia dal dottor Joseph Clover, "il più esperto cloroformista del momento". Il sondaggio confermò la presenza di un calcolo "grosso come un uovo di piccione", e venne subito pianificato un intervento di litotrissia.

Il paziente rientrò momentaneamente nella sua residenza, per essere poi operato il 2 gennaio (1873), sempre in anestesia cloroformica (anche questa volta praticata da Clover). Localizzato, il calcolo (di fosfati) venne schiacciato e parte dei detriti asportata. Ma nei due giorni seguenti, la frequente urinazione, il dolore e l'emorragia indicarono che un grosso frammento si era fermato nell'uretra posteriore; e il 6 gennaio, ancora sotto anestesia, esso fu sospinto in vescica e frantumato.

Immediatamente comparvero però altri segni di ostruzione. Fu così pianificato un terzo intervento, per il 9 gennaio. Ma lo stesso mattino il paziente entrò in coma uremico e morì in serata, alla presenza della moglie Eugene e del figlio (che poco dopo morirà nella guerra nello Zululand).

L'autopsia fu eseguita dal celebre John Burdon-Sanderson: i reni si presentavano ipertrofici, con segni di idronefrosi; la vescica conteneva un mezzo calcolo del 
peso di $7 \mathrm{~g}$ e del diametro di circa $3,5 \mathrm{~cm}$.

Al chirurgo (Henry Thompson) che aveva eseguito l'intervento fu offerta una somma di 2000 sterline, ma egli insistette per accettarne soltanto la metà. In fondo se lo poteva permettere, essendo il più quotato urologo europeo e vantando clienti di alto rango come Leopoldo I del Belgio.

Chi oggi si reca a Londra e vuol visitare l' Hunterian Museum of the Royal College of Surgeons, poco distante dal British Museum, può ammirare i frammenti del calcolo fosfatico estratti all'ex imperatore francese. Peccato che non possa ammirare gli oltre mille calcoli asportati nella sua brillante carriera da Thompson e che rappresentavano un vanto del Museo stesso: purtroppo andarono irrimediabilmente perduti insieme ad altre preziose raccolte, durante un bombardamento di Londra del 1941. In compenso, è ancora conservata la pinza impiegata da Thompson per l'estrazione: basta uno sguardo per apprezzare ancora una volta quali enormi passi siano stati compiuti da allora, nel breve volgere di un secolo, nel trattamento chirurgico della nefrolitiasi; e soprattutto per rendersi conto di quanto poco divertente sarebbe oggi sottoporsi ad interventi del genere con l'anestesia, i "ferri" e le tecniche di allora, anche se ci assicurassero che siamo nelle mani del più famoso urologo del tempo com'era Henry Thompson...

Indirizzo degli Autori:

Professor Luciano Sterpellone 1.sterpellone@libero.it 